\title{
Ray Tracing Analysis of Light Scattering Properties of Randomly Nano-Textured ZnO Films
}

\author{
M. Schulte ${ }^{\mathrm{a}}$, K. Bittkau ${ }^{\mathrm{a}}$, B. E. Pieters ${ }^{\mathrm{a}}$, S. Jorke ${ }^{\mathrm{a}}$, H. Stiebig ${ }^{\mathrm{b}}$, J. Hüpkes ${ }^{\mathrm{a}}$, and U. Rau ${ }^{\mathrm{a}}$ \\ ${ }^{a}$ IEF5-Photovoltaik, Forschungszentrum Jülich GmbH, 52425 Jülich, Germany \\ ${ }^{\mathrm{b}}$ Malibu GmbH \& Co. KG, Böttcherstraße 7, 33609 Bielefeld, Germany
}

\begin{abstract}
We investigate the scattering behavior of nano-textured $\mathrm{ZnO}$-Air and $\mathrm{ZnO}$-Silicon interfaces for the application in thin film silicon solar cells. Contrary to the common approach, the numerical solution of the Maxwell's equations, we introduce a ray tracing approach based on geometric optics and the measured interface topography. The validity of this model is discussed by means of SNOM measurements and numerical solutions of the Maxwell's equations. We show, that the ray tracing model can qualitatively describe the formation of micro lenses, which are the dominant feature of the local scattering properties of the investigated interfaces. A quantitative analysis for the ZnO-Silicon interface at $\lambda=488$ $\mathrm{nm}$ shows that the ray tracing model corresponds well to the numerical solution of the Maxwell's equations. At $\lambda=780$ $\mathrm{nm}$, a good agreement up to distance of approximately $1.5 \mu \mathrm{m}$ from the topography minimum is achieved. The reduced effective wavelength in silicon leads to a better description of the $\mathrm{ZnO}$-Silicon interface with respect to the $\mathrm{ZnO}$-Air interface by the ray tracing model.
\end{abstract}

Keywords: solar cell, TCO, ZnO, light trapping, light scattering, SNOM

\section{INTRODUCTION}

For amorphous and microcrystalline thin-film silicon solar cells, transparent conducting oxides (TCO) such as aluminum-doped zinc oxide $(\mathrm{ZnO})$ are commonly used as front contact [1-3]. The optimization of light trapping, induced by the texture of the TCO front contact, is a current topic of interest [4-11]. To understand the local influence of single TCO surface features on the light scattering and light trapping properties, correlations between texture topography and its scattering behavior have to be found.

A common approach for the description of light propagation at nano-textured interfaces, consists of numerically solving the Maxwell's equations [4-6]. However, from the final results of such a solution, the influence of single structural surface features on the scattering behavior is difficult to extract. In addition, it is sometimes difficult to distinguish various effects, such as distinguishing the lightening rod effect from a focusing effect caused by the surface profile [5].

Therefore, we introduce a ray tracing approach based on geometric optics and the measured TCO surface topography. Geometric optics provides a good approximation, if structural feature sizes are larger than the order of magnitude of the wavelength. This condition is not met for textured TCO surfaces applied in solar cells and the influence of diffractive effects (interference and deflection) on its optical properties is important. Consequently, e.g. near-field effects that are strongly related to the wave nature of light, cannot be described by the ray tracing model.

In this article, we analyze the scattering behavior of a textured $\mathrm{ZnO}$-Air and $\mathrm{ZnO}$-Silicon interface by means of the ray tracing model. To investigate the validity of the ray tracing approach, we compare our results to a numerical solution of the Maxwell's equations and, in the case of a ZnO-Air interface, also to scanning near-field optical microscopy (SNOM) measurements.

*me.schulte@fz-juelich..de, phone +49 2461 61-1553; fax +49 2461 61-1553; www.fz-juelich.de/ief/ief-5 
In Section 2, the sample preparation, the SNOM setup, and the sample topography is described. In Section 3 we introduce the ray tracing model. In Section 4, the ray tracing approach is discussed qualitatively for the TCO-Air interface in comparison to SNOM measurements from reference [5] and a numerically 3D solution of the Maxwell's equations from reference [5] based on the Finite Difference Time Domain (FDTD) method. In Section 5, the validity of the ray tracing model is checked for a $\mathrm{ZnO}$-Silicon interface by a comparison to a numerical solution in $2 \mathrm{D}$ of the Maxwell's equations using Chandezon's method [12,13]. Then, the model is applied in 3D to a ZnO-Silicon interface. Differences between the scattering properties of $\mathrm{ZnO}$-Air and $\mathrm{ZnO}$-Silicon interfaces and the application of the ray tracing model for both interfaces are discussed. Finally, the conclusions are presented in section 6 .

\section{EXPERIMENTAL}

\subsection{Sample Preparation}

The transparent conductive front electrode for the application in a solar cell is manufactured by magnetron sputtering of an approximately $800 \mathrm{~nm}$ thin film on a glass substrate. After the deposition process, the $\mathrm{ZnO}$ texture is achieved by a post etching step in hydrochloric acid solution. On this type of $\mathrm{ZnO}$ substrates solar cells with high efficiencies have been demonstrated [19].

\subsection{Scanning Near-field Optical Microscopy}

Scanning near-field optical microscopy (SNOM) is able to measure both, surface topography and local scattered intensities. Details of the experimental SNOM setup are described in [14,15].

The SNOM collection mode [16] allows for a microscopic study of local transmitted, scattered light [17]. Fig. 1 (a) shows a schematic view of the collection mode geometry. The specimen is illuminated from the glass side, while the transmitted, scattered intensity is collected by a tapered fiber tip. The transmitted intensity can be detected at different positions $(x, y)$ and different heights $(z)$ from the surface.

Fig. 1 (b) shows an image of the investigated $\mathrm{ZnO}$ surface determined from the SNOM topography mode.
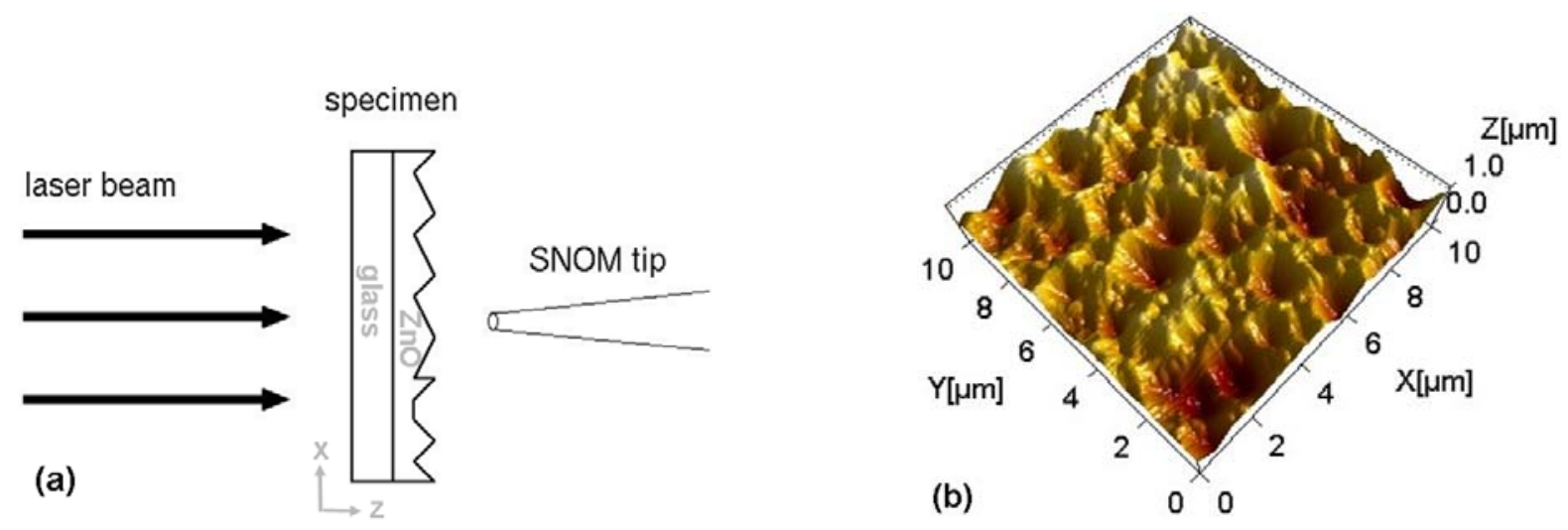

Figure 1: Schematic view of the SNOM experiment (a). The specimen is illuminated from the glass side. The transmitted, scattered intensity is collected by the fiber tip. (b) Topography of the investigated $\mathrm{ZnO}$ surface measured by the SNOM topography mode. 


\section{RAY TRACING}

\subsection{The Model}

In the ray tracing model, light propagates along straight lines. At a textured interface, the parallel incident light rays are scattered. The scattering angle $\theta_{\text {Medium } 1}$ is determined by using the incident angle $\theta_{\text {Medium 2, }}$, refractive indices $n_{\text {Medium } 1}$ and $\mathrm{n}_{\text {Medium } 2}$ from both media, and applying geometric optics, i.e. Snell's law

$$
\mathrm{n}_{\text {Medium } 1} \sin \left(\theta_{\text {Medium 1 }}\right)=\mathrm{n}_{\text {Medium } 2} \sin \left(\theta_{\text {Medium 2 }}\right)
$$

The principle of this approach applied to a textured $\mathrm{ZnO}$-Air interface is schematically explained in 2 dimensions in Fig. 2. Transmission and reflection at the interface are determined from a combination of the Fresnel coefficients and a Monte Carlo step. The absorption is determined from the incident intensity $\mathrm{I}_{0}$, light path length $\mathrm{d}$ and the absorption coefficient $\alpha$ by using Lambert-Beer's law

$$
\mathrm{I}(\mathrm{z})=I_{0} \cdot \exp (-\alpha \cdot d)
$$

The local intensity corresponds to the sum of intensities of those light rays, that intersect the interval $\Delta x$ (2D) or an area of $\Delta \mathrm{x}^{2}$ (3D) at a certain height $\mathrm{z}_{0}$. This interval/area corresponds to the aperture of the SNOM tip in the experiment. In the simulations we use the interface topography of the $\mathrm{ZnO}$ surface as it is determined during the SNOM measurements. The wavelength is only taken into account by refractive index, absorption coefficient, and the appropriate aperture length $\Delta \mathrm{x}$. These parameters are listed in Table 1. The abbreviation $\mu \mathrm{c}-\mathrm{Si}: \mathrm{H}$ stays for hydrogenated microcrystalline silicon.

Considering light as electromagnetic waves, intensity maxima and minima result from the interference of propagating waves. The distance between two neighboring intensity maxima cannot be lower than $\lambda / 2$ depending on the scattering angles. The lateral sizes of isolated intensity peaks are, therefore, larger than the resolution of the experimental SNOM tip and related to the wavelength. To this end, in the following ray tracing simulations we set the aperture length $\Delta x=\lambda_{\text {eff }}$ /2. The effective wavelength is the wavelength in the medium in which it is propagating and defined here as $\lambda_{\text {eff }}=\lambda_{\text {Air }}$ $/ \mathrm{n}_{\text {Medium }}$.

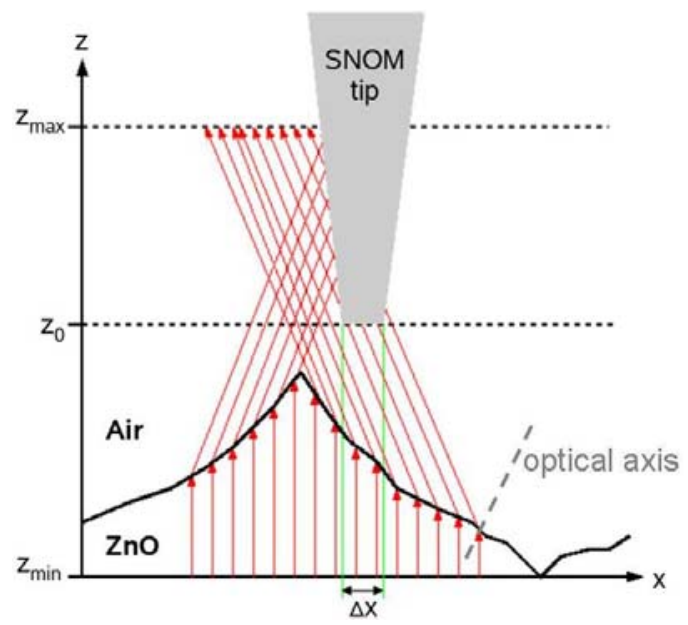

Figure 2: Schematic illustration of the ray tracing model for a ZnO-Air interface. The red arrows indicate the light paths. As light rays intersect the $\mathrm{ZnO}$-Air interface, they are refracted away from the optical axis.

Table 1. Simulation parameters for zinc oxide, air and hydrogenated microcrystalline silicon: refractive index $\mathrm{n}$, absorption coefficient $\alpha$, effective wavelength $\lambda_{\text {eff }}$, and aperture length $\Delta \mathrm{x}$ for the wavelengths $\lambda=488 \mathrm{~nm}$ and $780 \mathrm{~nm}$.

SIMULATION PARAMETERS

\begin{tabular}{cccccc} 
& $\boldsymbol{\lambda}[\mathbf{n m}]$ & $\mathbf{n}$ & $\boldsymbol{\alpha}\left[\mathbf{c m}^{-\mathbf{1}}\right]$ & $\boldsymbol{\lambda}_{\text {eff }}[\mathbf{n m}]$ & $\boldsymbol{\Delta} \mathbf{x}[\mathbf{n m}]$ \\
\hline \multirow{2}{*}{ ZnO } & 488 & 2.0 & 0.0 & 244 & - \\
& 780 & 2.0 & 0.0 & 390 & - \\
\multirow{3}{*}{ Air } & 488 & 1.0 & 0.0 & 488 & 244 \\
& 780 & 1.0 & 0.0 & 780 & 400 \\
$\boldsymbol{\mu c - S i : H}$ & 488 & 4.0 & 48569.7 & 122 & 61 \\
& 780 & 3.4 & 751.3 & 229 & 115
\end{tabular}




\section{SCATTERING BEHAVIOR FOR A ZNO-AIR INTERFACE}

\subsection{Results}

Fig. 3 (a) and 3 (b) show the measured and simulated height intensity profile of transmitted light at a ZnO-Air interface, respectively, both taken from reference [5]. The measured intensity distribution shown in Fig. 3 (a) has a height resolution of $350 \mathrm{~nm}$. The image pixels in between have been interpolated. This interpolation method is explained in reference [15]. Fig. 3 (b) shows the result obtained from Finite-Difference Time-Domain (FDTD) simulations, whereas Fig. 3 (c) shows the result determined from the ray tracing approach. In Fig. 5 (a) and 5 (b) the measured SNOM intensities from reference [5], 3D ray tracing intensities of a whole $10 \times 10 \mu \mathrm{m}^{2}$ scan and the investigated surface topography can be seen. These intensities are shown for $\lambda=488 \mathrm{~nm}$ and a distance $\Delta \mathrm{z}=1250 \mathrm{~nm}$ from the minimum point of the surface topography.

In Fig. 3 (a) local maxima and minima in the measured SNOM intensities can be observed. Up to a height of $\mathrm{z} \approx 3 \mu \mathrm{m}$ the local maxima and minima in intensity are located primarily above the respective maxima and minima of the topography. At heights above $3 \mu \mathrm{m}$ the intensity maxima are no longer directly correlated to the topography height. Fig. 5 (a) shows the whole measured surface area at $\mathrm{z}=1250 \mathrm{~nm}$. We observe a ring-like structure of the intensity distribution. Varying the wavelength in the range of 488-780 $\mathrm{nm}$ does not significantly influence the shape of the intensity maxima: The ring-like maxima are slightly broadened with increasing wavelength (not shown, see reference [5]).

The solution of the FDTD in Fig. 3 (b) shows relatively sharply confined intensity maxima and minima. In comparison to the SNOM measurement in Fig. 3 (a), the position of the brightest maxima and minima is relatively well reproduced within the simulation. Due to the low resolution in $\mathrm{z}$ direction and the interpolation process, near-field effects which occur at the first $500 \mathrm{~nm}$ above the surface are not visible in Fig. 3 (a).

Below a height of $z \approx 3 \mu \mathrm{m}$, the calculated ray tracing intensities in Fig. 3 (c) show the formation of intensity maxima (minima) over of the topography maxima (minima). This is in good agreement with measured intensities and intensities determined from the FDTD simulation. Above a height of approximately $3 \mu \mathrm{m}$, a diffuse distribution of the scattered light is found without local intensity maxima and minima, which cannot be seen in the measurement and FDTD simulation. Near-field effects which can be observed in Fig. 3 (b) are also not reproduced in the ray tracing approach.

In Fig. 5 (b), the intensity distribution determined by the ray tracing model of the whole investigated surface area at $\mathrm{z}=1250 \mathrm{~nm}$ is shown. We observe a ring-like structure of the intensity distribution. The measured ring-like distribution shown in Fig. 5 (a) is well reproduced within the ray tracing simulation shown in Fig. 5 (b).

\subsection{Discussion}

A comparison of the brightest intensity maxima (shown in Fig. 3 (a) and 3 (c)) below $z=3 \mu \mathrm{m}$ to the measured surface topography (Fig. 1 (b)) shows, that intensity foci close to the surface can be correlated to the position of the crater rims in the topography, while low intensities are rather located in the crater centers. This explains also the ring-like intensity distributions from Fig. 5 (a) and 5 (b).

Near-field effects that are shown in the FDTD simulation are contributed to evanescent waves, which are discussed in reference [18] in more detail. These evanescent waves decrease exponentially with the distance from the surface and therefore only have an impact on the intensities directly above the surface up to a height of $0.3 \mu \mathrm{m}$ [18]. As such nearfield effects are strongly related to the wave nature of light, they cannot be explained by the ray tracing model. It should be noted, that the height resolution of the presented SNOM measurement is not satisfactory for resolving near field effects.

In Ref. [5], the origin of the intensity foci were discussed to be related to the lightening rod effect or to a focusing effect by the parabolic surface profile. The lightening rod effect causes the enhancement of near-fields close to sides where the surface curvature of micro- and nanometer sized particles is the largest. The lightening rod effect cannot be described by the ray tracing model. But the formation of light foci is described by means of the ray racing model as a micro lens effect without a need of additional effects, such as the lightening rod effect. 

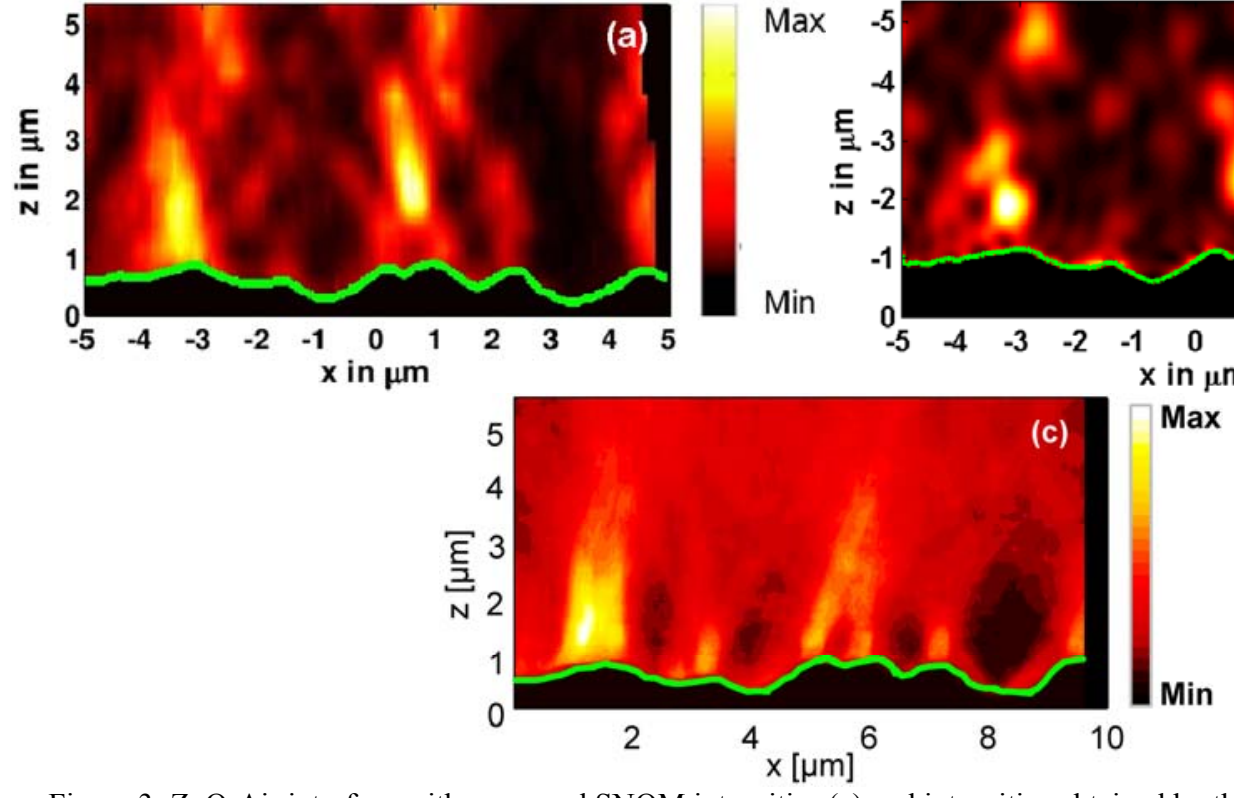

Figure 3: ZnO-Air interface with measured SNOM intensities (a) and intensities obtained by the 3D solution of the

Maxwell's equations (b) both from Ref. [5], and calculated 3D Ray tracing intensities (c) with aperture size $\Delta x=400$ $\mathrm{nm}$. The investigated wavelength $\lambda$ is $780 \mathrm{~nm}$. The green line indicates the appropriate $\mathrm{ZnO}$ surface profile, which is part of the topography shown in Fig. 1 (b).

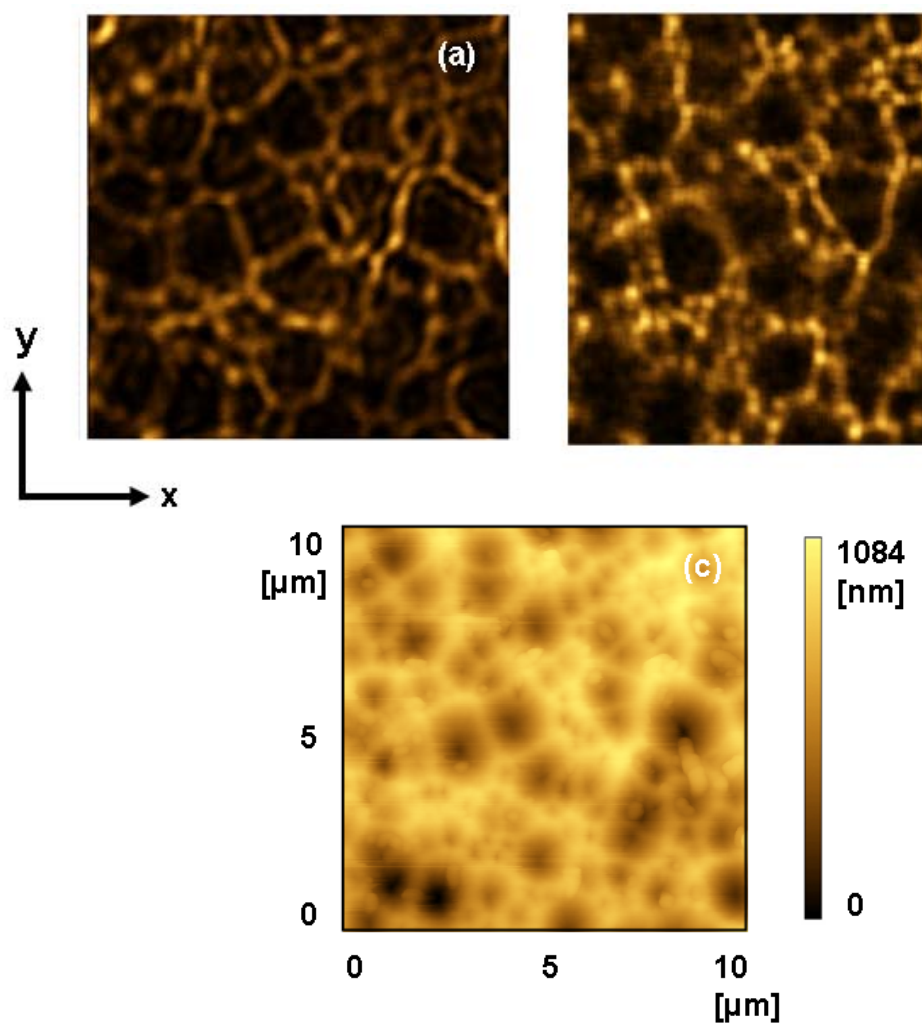

Figure 4: Measured SNOM intensities at the ZnO-Air interface (a) from Ref. [5] and 3D ray tracing intensities at the $\mathrm{ZnO}$ Air interface (b) of a $10 \times 10 \mu \mathrm{m}^{2}$ scan at a distance $\Delta \mathrm{z}=1250 \mathrm{~nm}$ from the minimum point of the surface topography with aperture length $\Delta x=244 \mathrm{~nm}$ and $\lambda=488 \mathrm{~nm}$. Intensity peaks are observed to be located above the crater rims of the surface topography (c), which is identical to the topography in Fig. 1 (b). 

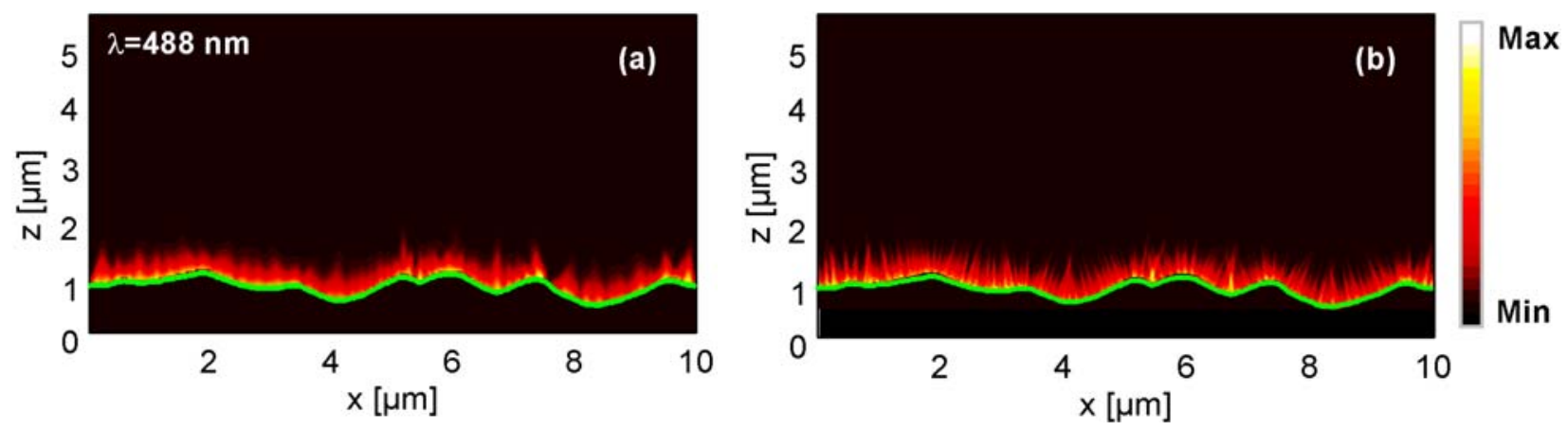

Figure 5: ZnO-Silicon interface with calculated 2D intensities at $\lambda=488 \mathrm{~nm}$ by (a) the solution of the Maxwell's equations using Chandezon' s method and (b) the ray tracing model with aperture size $\Delta x=61 \mathrm{~nm}$. The green line indicates the $\mathrm{ZnO} 2 \mathrm{D}$ surface profile, which is part of the 3D topography shown in Fig. 1 (b).
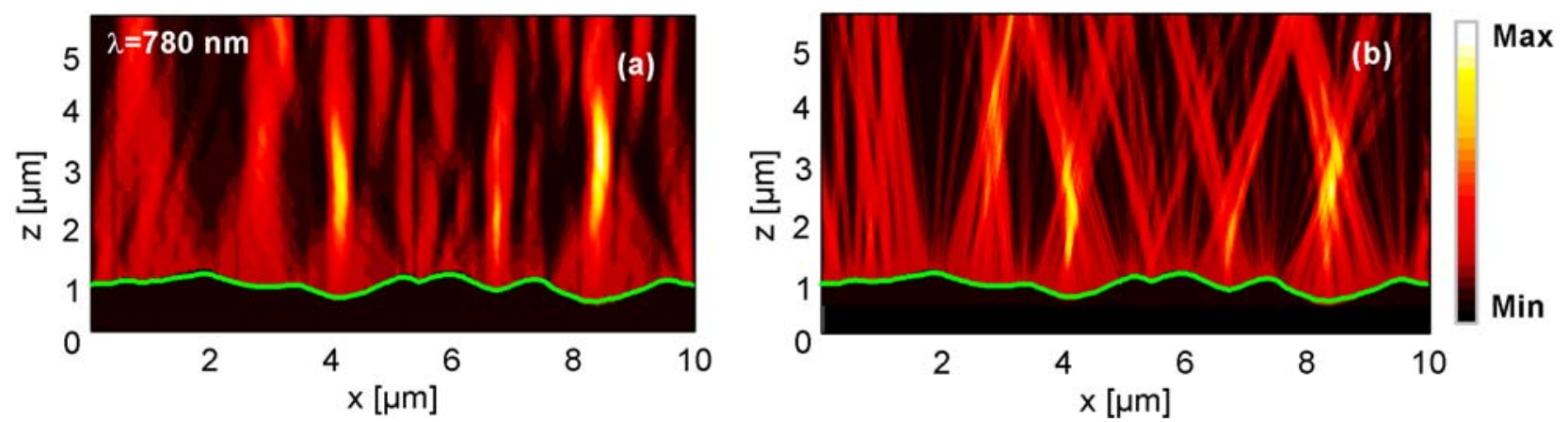

Figure 6: $\mathrm{ZnO}$-Silicon interface with calculated 2D intensities at $\lambda=780 \mathrm{~nm}$ by (a) the solution of the Maxwell's equations using Chandezon's method and (b) the ray tracing model with aperture size $\Delta x=\lambda_{\text {silicon }} / 2=115 \mathrm{~nm}$. The green line indicates the $\mathrm{ZnO} 2 \mathrm{D}$ surface profile, which is part of the 3D topography shown in Fig. 1 (b).
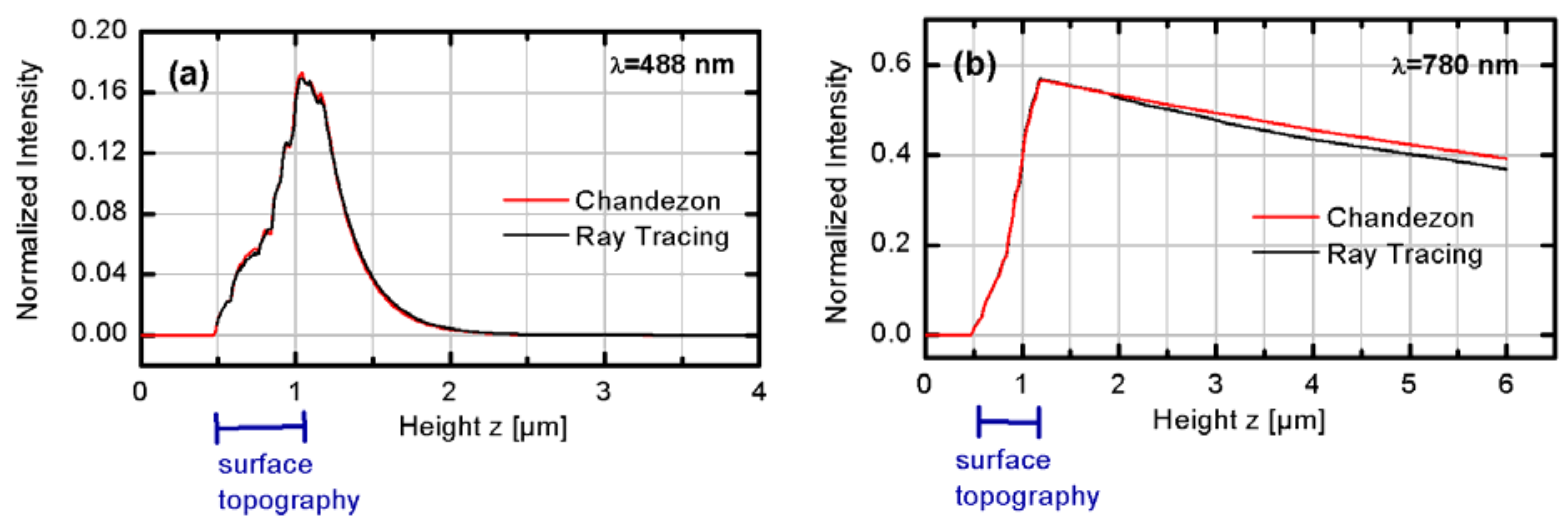

Figure 7: Comparison of the intensities at the $\mathrm{ZnO}$-Silicon interface for Chandezon's method and the ray tracing approach, determined from Figs. 5 and 6 (a) for $\lambda=488 \mathrm{~nm}$ and (b) $\lambda=780 \mathrm{~nm}$. The intensity is normalized to the incident intensity. 

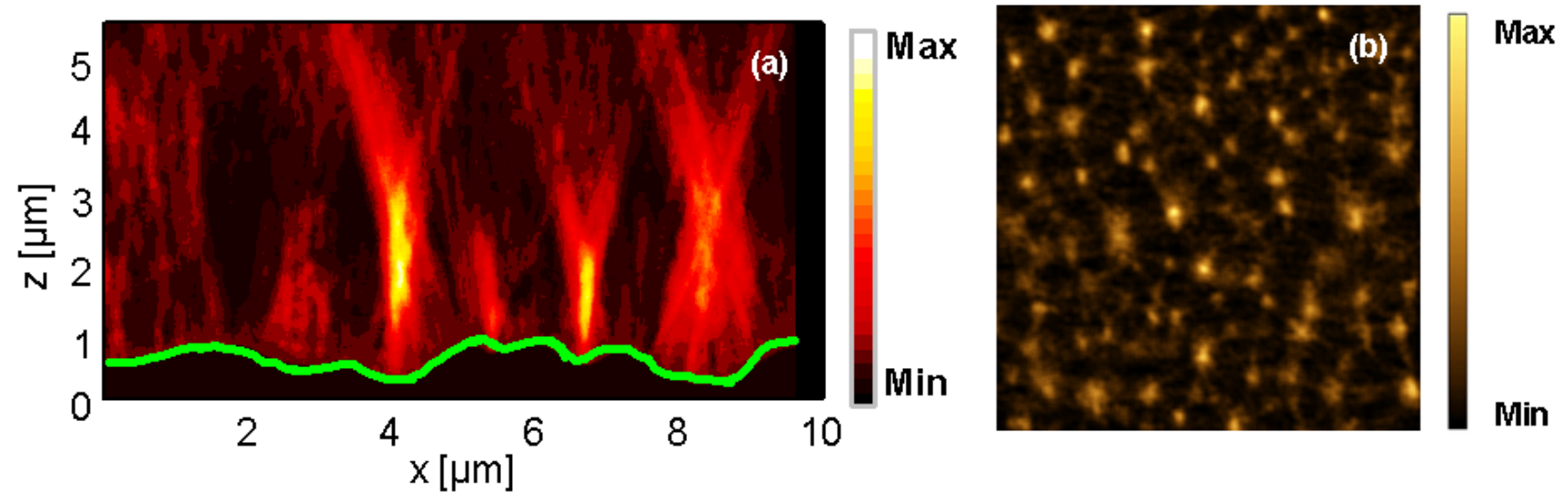

Figure 8: 3D ray tracing intensities at the $\mathrm{ZnO}$-Silicon interface (a) for different heights and (b) at a distance $\Delta \mathrm{z}=2050 \mathrm{~nm}$ from the minimum point of the surface topography for a $10 \times 10 \mu \mathrm{m}^{2}$ scan with aperture length $\Delta \mathrm{x}=\lambda_{\text {Silicon }} / 2=115 \mathrm{~nm}$ and $\lambda=780 \mathrm{~nm}$. The green line indicates the appropriate $\mathrm{ZnO}$ surface profile, which is part of the topography shown in Fig. 1 (b). Intensity peaks are observed to be located above the crater centers of the surface topography (see Fig. 5 (c)).

In the ray tracing model, light rays are bundled over the position of the crater rims as they are refracted away from the optical axis. The rays from two neighboring craters intersect and result in intensity foci. Therefore, in crater centers small intensities are observed. Fig. 9 (a) shows the principle of focus formation in air by means of the ray tracing model. The focus size $\left(\Delta \mathrm{z}_{\mathrm{f}}, \Delta \mathrm{x}_{\mathrm{f}}\right)$ depends on the one hand on the diameters of the neighboring craters and on the other hand it is influenced by the direction of the scattered light rays. Since geometric optics is applied in the model, the direction of the scattered light rays is determined from the incident angle at the $\mathrm{ZnO}$-Air interface and refraction indices of both media.

The ray tracing approach can explain the main features of the local scattering properties, i.e. the formation of micro lenses at distances from the surface below $3 \mu \mathrm{m}$. Above a height of $3 \mu \mathrm{m}$ deviations between the measured SNOM intensities and the ray tracing intensities are observed. The optical scattering properties above $3 \mu \mathrm{m}$ are dominated by diffractive optics, which include the effects interference and deflection.

Our analysis shows, that for the considered surface and wavelengths the scattering properties are described by an overlay of refractive (geometric optics) and diffractive (deflection and interference) optics. The important feature of micro lensing at the crater rims is explained by the refractive part, whereas e.g. near-field effects are contributed to diffractive optics.

\section{SCATTERING BEHAVIOR FOR A ZNO-SILICON INTERFACE}

\subsection{Motivation and Method}

For thin film silicon solar cells, the scattering properties of a $\mathrm{ZnO}$-Silicon interface are relevant. Therefore, the scattering properties of a $\mathrm{ZnO}$-Silicon interface are considered. In this case the results of the ray tracing model cannot be compared directly to experiments as we cannot measure the light intensity within a silicon layer with SNOM. For this reason we first compare the ray tracing method to numerical solutions of the Maxwell's equations obtained with Chandezon's method.

Chandezon's method can solve the Maxwell's equations in two dimensions. For the validation of our ray tracing model with Chandezon's method we assume a $16 \mu \mathrm{m}$ thick $\mu \mathrm{c}-\mathrm{Si}: \mathrm{H}$ layer on top of a $\mathrm{ZnO}$ half-space with a subsequent non absorbing layer of the same refractive index as the $\mu \mathrm{c}-\mathrm{Si}: \mathrm{H}$ layer. For the interface between the $\mathrm{ZnO}$ and the $\mu \mathrm{c}-\mathrm{Si}: \mathrm{H}$ we used a single topography line of the texture etched $\mathrm{ZnO}$ substrate. In the simulations using the ray tracing model both the $\mathrm{ZnO}$ and the $\mu \mathrm{c}-\mathrm{Si}: \mathrm{H}$ consist of half spaces.

After a validation of the ray tracing model in $2 \mathrm{D}$, simulations for a $3 \mathrm{D}$ texture, as it is implemented in thin film silicon solar cells, are performed by using the topography shown in Fig. 1 (b). 


\subsection{Comparison of Ray Tracing and Chandezon in 2D}

Fig. 5 shows both simulations of the ray tracing approach and of Chandezon's method for $\lambda=488 \mathrm{~nm}$. We observe a strong decrease of the intensity with increasing height. No intensity foci are obtained for this wavelength. A comparison of Chandezon's method and the ray tracing approach shows a good agreement of both simulations. Fig. 6 shows both simulations of the ray tracing approach and of Chandezon's method for $\lambda=780 \mathrm{~nm}$. Intensity foci for both types of simulation are achieved. We observe intensity foci similar to the case of a $\mathrm{ZnO}$-Air interface (see Figs. 3 and 4). However, contrary to the $\mathrm{ZnO}$-Air interface, the intensity foci are particularly found above the topography minima and not above topography maxima. In addition, intensity foci in silicon are clearly narrower than intensity foci in air. The focus points are observed at a height $\mathrm{z}$ of approximately $2 \mu \mathrm{m}$. If we compare Fig. 6 (a) and 6 (b), the brightest intensity maxima in Chandezon's method are well reproduced with the ray tracing simulation. Beside these intensity maxima up to a height of approximately $3.5 \mu \mathrm{m}$, also single details in the scattered intensities, such as cone shaped intensity distributions over the surface, are well reproduced with the ray tracing approach. Above a height of $z \approx 3.5 \mu \mathrm{m}$, deviations in the intensity distributions are observed.

For a quantitative comparison of Chandezon's method and the ray tracing approach the mean intensity as a function of height $\mathrm{z}$ for $\lambda=488 \mathrm{~nm}$ and $\lambda=780 \mathrm{~nm}$ is chosen as a figure of merit. The results are shown in Fig. 7 (a) and 7 (b). We note here, that for the investigated topography, the distance between maximum and minimum $\mathrm{z}$ value of the topography is approximately $1.1 \mu \mathrm{m}$. Due to the increasing volume fraction consisting of silicon with increasing height, an increase of the intensity with increasing height is achieved.

For $\lambda=488 \mathrm{~nm}$, both simulations correspond very well. For $\lambda=780 \mathrm{~nm}$, we observe a good correspondence up to a height of approximately $\mathrm{z} \approx 2 \mu \mathrm{m}$. This corresponds to a distance of $1.5 \mu \mathrm{m}$ to the minimal $\mathrm{z}$ value of the surface topography. Above this limit, deviations in the intensities between both simulations are observed: The intensity for Chandezon's method is clearly higher than the intensity determined by the ray tracing model. A comparison between $\lambda=488 \mathrm{~nm}$ and $\lambda=780 \mathrm{~nm}$ shows for both simulations a steeper decrease of the intensity as a function of $\mathrm{z}$ for $\lambda=488 \mathrm{~nm}$.

In Fig. 5 (a) and Fig. 6 (a) we do not observe near-field effects in the silicon layer, which can clearly be observed in Fig. 3 (b) in air. An analysis shows, that no evanescent waves are determined from Chandezon's method (not shown). This might be also related to restrictions in the method when simulating a large spatial domain with a high refractive absorbing material. As near-field effects cannot be described by the ray tracing model, a better description of the $\mathrm{ZnO}$ Silicon interface with respect to the $\mathrm{ZnO}$-Air interface by the ray tracing model is achieved. This is explained by the three times shorter effective wavelength in the silicon compared to air (see Table 1). The surface features are thus effectively larger in silicon compared to the wavelength in air, making geometric optics more suitable to describe $\mathrm{ZnO}$ Silicon interfaces than $\mathrm{ZnO}$-Air interfaces.

\subsection{Results in 3D}

To calculate the scattering intensities of a real interface topography, 3D simulations for the surface are carried out and shown in Fig. 8. In particular the light scattering properties in the long wavelength range are interesting for the light trapping due to the low absorption coefficient of the silicon in this wavelength range. Therefore, we consider exemplarily a wavelength $\lambda=780 \mathrm{~nm}$. The simulation results are shown in Fig. 8 (a) and 8 (b).

The calculated intensities shown in Fig. 8 (a) resemble the intensities obtained from the 2D simulation shown in Fig. 6 (b). The intensity maxima are observed approximately at the same heights (around a distance of around $\mathrm{z} \approx 2 \mu \mathrm{m}$ from the minimum of the 3D surface topography). Compared to Fig. 6 (b) some intensity maxima in Fig. 8 (a) are attenuated and some are amplified.

Fig. 8 (b) shows a top view at a distance of $z=2050 \mathrm{~nm}$ from the minimum of the surface topography. The foci at this distance are typically point-shaped and do not show the ring-like structure as in air (see Fig. 5). 


\subsection{Analysis and Discussion}

The formation of intensity maxima in silicon for $\lambda=780 \mathrm{~nm}$ is described by the ray tracing approach. Fig. 9 explains schematically, why intensity foci are found in air above the topography maxima and in silicon above topography minima. In air, light rays are refracted away from the optical axis and intersect over the topography maxima. At the intersection area, a higher intensity is determined. In silicon, light rays are refracted in direction of the optical axis as light propagates at the interface into an optically denser material. The intersection area is located over the topography minima. For $\lambda=488 \mathrm{~nm}$ no intensity foci are observed. This can be explained by the high absorption coefficient of the silicon for this wavelength: Light rays are absorbed before they can intersect. This becomes clearer when comparing the intensity decrease with $\mathrm{z}$ at $\lambda=488 \mathrm{~nm}$ and $\lambda=780 \mathrm{~nm}$ in Fig. 7 (a) and 7 (b) again. At the surface topography maximum, the intensity is decreased to approximately 0.15 for $\lambda=488 \mathrm{~nm}$, while for $\lambda=780 \mathrm{~nm}$ the intensity is approximately 0.6 . As intensity decreases exponentially with the light path, light rays are absorbed before they reach a possible intersection point of approximately one micrometer further. Indeed, the intensity profile shown in Fig. 7 (a) has decreased to 0.004 at $\mathrm{z}=2 \mu \mathrm{m}$.
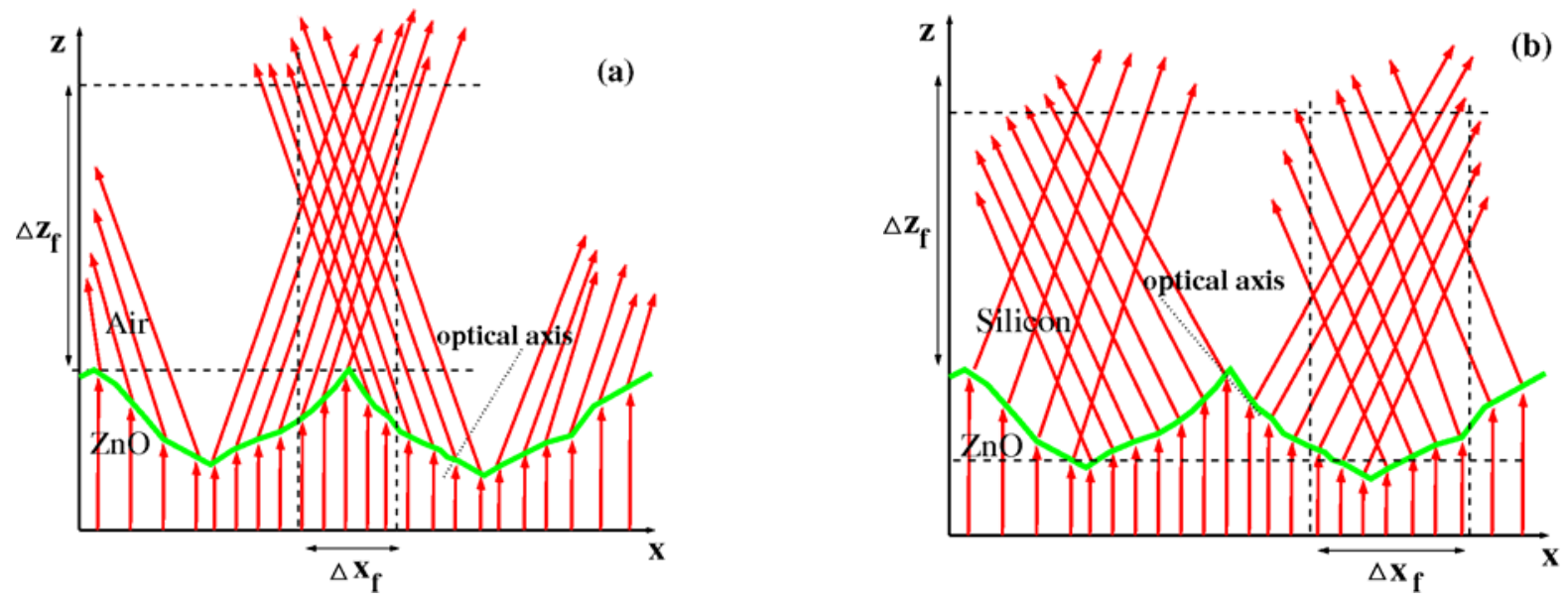

Figure 9: Schematic explanation of the micro lens effect (a) at a $\mathrm{ZnO}$-Air interface and (b) a ZnO-Silicon interface with a low absorption in the silicon. In case of the $\mathrm{ZnO}$-Air interface, light rays are refracted in direction of the crater edges and intersect. At the $\mathrm{ZnO}$-Silicon interface, rays intersect at the position of the crater centers. Please note that light rays are shown only schematically and usually do not propagate parallel to each other.

From an analysis of the 3D surface topography arises, that intensity foci shown in Fig. 8 are located right above the crater centers. This is contrary to the observations in air, where the intensity maxima are located above the crater rims. Deviations between simulations of Chandezon's method and the ray tracing model have been quantified by means of the average intensity as a function of height $\mathrm{z}$ (see Fig. 7). For $\lambda=780 \mathrm{~nm}$ we can see, that both simulations clearly differ from each other for approximately $\mathrm{z}>2 \mu \mathrm{m}$. This can be explained by an overestimation of scattering angles by the ray tracing approach. This explanation appears reasonable, as for nano-textured surfaces specularly scattered light, i.e. light scattered into an angle of $0^{\circ}$, has been already observed and discussed $[20,21]$. The ray tracing approach does not describe specularly scattered light, because almost no surface angles (incident angles $\theta_{\text {Medium }}$ ) at $0^{\circ}$ are found for the investigated surface (see Eqn. 1). However, although the trend of the overestimation of scattering angles by the ray tracing approach will remain, scattering angles can also be underestimated by Chandezon's method due to a limited number of Fourier components.

\section{CONCLUSIONS}

We investigated the scattering behavior of nano-textured $\mathrm{ZnO}$-Air and $\mathrm{ZnO}$-Silicon interfaces. To this end we developed a ray tracing model which we compared to SNOM measurements and numerical solutions of the Maxwell's equations. We could show, that the ray tracing model can qualitatively describe the main features of the scattering properties, i.e. 
micro lensing effects, for the $\mathrm{ZnO}$-Air as well as for the $\mathrm{ZnO}$-Silicon interface. Furthermore, the position of micro lenses can be directly correlated to the investigated crater-like interface structure: Due to the different refractive indices of air and silicon for $\lambda=780 \mathrm{~nm}$, light rays are refracted in different directions with respect to the optical axis and bundled over crater rims in the case of the $\mathrm{ZnO}$-Air interface and over crater centers in case of the $\mathrm{ZnO}$-Silicon interface.

A quantitative analysis of the intensities at the $\mathrm{ZnO}$-Silicon interface shows, that the ray tracing model is a good approximation for $\lambda=488 \mathrm{~nm}$. For $\lambda=780 \mathrm{~nm}$ a good correspondence is achieved for a distance of approximately $1.5 \mu \mathrm{m}$ from the topography minimum. The difference between the simulations of the ray tracing approach and Chandezon's method are caused due to an overestimation of the scattering angles by the ray tracing approach.

Contrary to the $\mathrm{ZnO}$-Air interface, the numerical solutions of the Maxwell's equations for the ZnO-Silicon interface do not show near-field effects. Therefore, a better description of the $\mathrm{ZnO}$-Silicon interface with respect to the ZnO-Air interface by the ray tracing model is achieved. This is explained by the more than three times shorter effective wavelength in the silicon compared to air. The surface features are thus effectively larger in silicon compared to the wavelength in air, making geometric optics more suitable to describe $\mathrm{ZnO}$-Silicon interfaces than $\mathrm{ZnO}$-Air interfaces.

\section{ACKNOWLEDGEMENTS}

We thank Thomas Beckers and Reinhard Carius for the fruitful discussions, Carsten Rockstuhl for his cooperation and Veeco for providing the SEM image of the SNOM probe. Financial support by the BMU (project number 0327625) is gratefully acknowledged.

\section{REFERENCES}

1. J. A. A. Selvan, Yuan-Min Li Sheyu Guo, A. E. Delahoy, Proc. 31rst IEEE, Vol. I, 1492 (2005).

2. L. Feitknecht, J. Steinhauser, R. Schlüchter, S. Fä̈, D. Dominé, E. Vallat-Sauvin, F. Meillaud, C. Ballif, A Shah, Technical Digest of PVSEC-15, Shanghai (2005).

3. H. Schade, P. Lechner, R. Geyer, H. Stiebig, B. Rech, O. Kluth, Conference Record of the Thirty-first IEEE 2005, Vol. I, 1436 (2005).

4. C. Haase, H. Stiebig, Applied Physics Letters, 91, 6 (2007).

5. C. Rockstuhl, F. Lederer, K. Bittkau, R. Carius, Applied Physics Letters 91 (2007).

6. C. Rockstuhl, S. Fahr, F. Lederer, K. Bittkau, T. Beckers, R. Carius, Applied Physics Letters, 93, 6 (2008).

7. J. Krc, M. Zeman, O. Kluth, F. Smole, M. Topic, Thin solid films, 426, 296-304 (2003).

8. H. Schade, Z. E. Smith, Applied Optics, 24, 19 (1985).

9. G. Tao, PhD thesis, Technical University Delft (1994).

10. R. Brendel, Progress in Photovoltaics, 3, 1, 25 (1995).

11. S. Fahr, C. Rockstuhl, F. Lederer, Appl. Phys. Lett., 92, 171114 (2008).

12. G. Behme, A. Richter, M. Süptitz, Ch. Lienau, Rev. Sci. Instrum. 68, 3458 (1997).

13. K. Bittkau, T. Becker, S. Fahr, C. Rockstuhl, F. Lederer, and R. Carius, Nanoscale investigation of lighttrapping in a-Si:H solar cell structures with randomly textured interface, Physica Status Solidi A, 205, 2766 (2008).

14. J. Chandezon, M. T. Dupuis, G. Cornet, D. Maystre, "Multicoated Gratings - A Differential Formalism Applicable in the Entire Optical Region", Journal of the Optical Society of America, 72, 7, 839-846 (1982).

15. J. Chandezon, D. Maystre, G. Raoult, "A new theoretical method for Diffraction Gratings and Its Numerical Application", Journal of Optics-Nouvelle Revue D Optique, 11 4, 235-241 (1980).

16. Betzig E, Isaacson M, Lewis A, Applied Physics Letters, 51, 2088 (1987).

17. K. Bittkau, R. Carius, C. Lienau, Physical Review B 76, 035330 (2007).

18. K. Bittkau and R. Carius, "Near-field study of optical modes in randomly textured $\mathrm{ZnO}$ thin films", Superlattices and Microstructures, 42, 1-6, 47-51 (2007).

19. O. Kluth, A. Loffl, S. Wieder, C. Beneking, W. Appenzeller, L. Houben, B. Rech, H. Wagner, S. Hoffmann, R. Waser, J. A. A. Selvan, H. Keppner, Proceedings of the 26th IEEE Photovoltaic specialists conference, 715-718 (1997). 
20. J. Krc, M. Zeman, O. Kluth, F. Smole, M. Topic, "Light scattering properties of $\mathrm{SnO} 2$ and $\mathrm{ZnO}$ surface-textured substrates", Proc. 3rd world conference in Photovoltaic energy conversion, A-C, 1823-1826 (2003).

21. F. Leblanc, J. Perrin, J. Schmitt, "Numerical modeling of the optical properties of hydrogenated amorphous silicon based pin solar cells deposited on rough transparent conducting oxide substrates", Journal of Applied Physics, 75, 2, 1074-1087 (1994). 